\title{
Patients with carbapenem-resistant Enterobacteriaceae in emergency room; is this a real problem?
}

\author{
Matias C Salomão ${ }^{1}$, Maristela P Freire*,1 \& Anna Sara S Levin² \\ ${ }^{1}$ Infection Control Department, Hospital das Clınicas, Universidade de São Paulo, Brazil \\ ${ }^{2}$ Department of Infectious Diseases, Faculdade de Medicina, Universidade de São Paulo, Brazil \\ *Author for correspondence: Tel./Fax: +55 112661 6444; maristelapf@uol.com.br
}

\begin{abstract}
"The CRE-colonized patients in hospitals are the tip of the iceberg of a much larger problem. The fact that we can detect carbapenemases coming from community settings, from patients in the $E R$, food, animals and others sources, demonstrates that this issue is no longer restricted to the hospitals, but to a much broader transmission network."
\end{abstract}

First draft submitted: 21 September 2019; Accepted for publication: 13 December 2019; Published online: 15 January 2020

Keywords: carbapenem-resistant Enterobacteriaceae • community acquired infection • community multidrug resistant infection • community-onset infection • CRE control • CRE in agricultural settings • emergency room • environment contamination • horizontal transmission

\section{Dissemination of carbapenem resistance among Enterobacteriaceae}

Carbapenem-resistant Enterobacteriaceae (CRE) have been identified as public health threat of international concern due to the very limited therapeutic options and the rapid worldwide dissemination.

The main mechanism of carbapenem-resistant Enterobacteriaceae (CRE) resistance is through carbapenemase production. The most frequent known carbapenemase is Klebsiella pneumoniae carbapenamase (KPC), first reported in the mid-1990s in the USA. This enzyme, classified as Ambler class A, had a worldwide spread, and nowadays it is estimated that $\mathrm{KPC}$ is responsible for $71 \%$ of carbapenem resistance among Enterobacteriaceae. The KPC is codified by a plasmid-borne gene, bla $a_{\mathrm{KPC}}$, which increases its ability to disseminate within and between species [1].

Other types of carbapenemases are those classified as Ambler Class B (Verona imipenemase, Imipenemase and New Dehli metallobetalactamase) and Class D (OXA-48, OXA-181). The incidence of these other carbapenemases varies geographically, but their dissemination is more limited, and they are commonly related to outbreaks [2].

The international SENTRY study described increasing CRE rates: from $0.6 \%$ in $1997-2000$ to $2.9 \%$ in $2013-$ 2016 ( $\mathrm{p}<0.05$ ) with gradual increases of $0.8-0.9 \%$ per year since $2005-2008$. This increase was greater in Europe when compared with Asia and the USA, but less compared with Latin America, where the increase was 5.6\% [1].

\section{The transmission of carbapenem-resistant bacteria}

The acquisition of CRE can occur in two ways: patient-to-patient transmission or other kinds of horizontal transmission; and through emergence of resistance in a carbapenem-susceptible strain. Epidemiological findings and the low levels of core genome diversity suggest that patient-to-patient transmission is more important. Strategies to control CRE dissemination are focused on improving hand hygiene, detecting of carriage early, instituting contact precautions, cohorting patients with dedicated staff and improving environmental cleaning. In addition, decolonization protocols have been used without substantial results [3].

Hand hygiene improvement is a core intervention for controlling the dissemination of CRE. A mathematical model was successfully used in an ICU to predict compliance hand hygiene and contact precautions necessary to control CRE spread. In this particular study, it was interesting to observe that after achieving the goal of hand hygiene compliance, CRE colonization rates decreased, but CRE was never abolished, since colonized-patients continued to be admitted into ICU coming from other wards such as the emergency room (ER) [4]. This finding

Future 8 Medicine 
is particularly interesting as it stresses the fact that CRE occurs not only in the ICUs but also in patients coming from community, other hospitals and other wards.

\section{Interactions between community \& hospital-settings regarding CRE acquisition}

The emergence of carbapenem resistance also seems to occur in the environment and in agricultural settings. CRE was isolated from hospital sewage in Brazil, China and Spain. Furthermore, CRE was found in community sources of water in many countries such as Portugal, Brazil and Italy. A recent report described CRE in retail chicken meat in Egypt and in fresh vegetables and spices from Asia [5-9].

However, the impact of these findings on CRE dissemination is unclear. The relationship between CRE in the environment and the development of community-associated infection (CAI) by CRE has not yet been established.

An infection by CRE identified in patients on hospital admission can be classified as a community-onset infection (COI) when the patient has a history of multiple exposures to the healthcare system, even if he or she has been an outpatient long enough to not fit the classical definition of healthcare association. The infection can be classified as CAI when the patient has no history of previous exposure to the healthcare system. Often it is not possible to distinguish between COI and CAI, but it has been reported that up to $30 \%$ of patients with CRE infection on hospital admission have had no previous exposure to the healthcare system [10].

CAI-CRE is reported in many settings and countries with large variations in incidence; the proportion of CAI or COI-CRE has been reported to be from 0 to $29 \%$. Although this incidence is usually low it can represent a caveat for CRE control [10]. Moreover, the patient could be just colonized by CRE without any sign of infection and became a silent source of these multidrug resistant bacteria in the hospital.

\section{Screening for carbapenem resistance}

It has been shown that rapid identification and isolation of CRE carriers on hospital admission led to a significant reduction of nosocomial transmission of CRE [11]. Also, sharing the information of CRE colonized patients in a governmental database led to reduction of more than $20 \%$ of carriers [12].

Patients with COI-CRE are more easily identified when compared with patients with CAI. Patients with COI can be suspected based on well-known risk factors such as exposure to the healthcare system, or antimicrobial use. In this situation, even without previous diagnosis of CRE colonization, the patient may be adequately screened for CRE and put on contact precautions. Long-term care facilities are important and well described sources of CRE carriage. In some reports, the incidence of CRE and its transmission in long-term care facilities is higher than in acute care hospitals $[2,13]$. Another risk factor for CAI-CRE is recent travel to areas where CRE is endemic.

The potential risk for CRE dissemination by an unrecognized carrier will depend in part on where these patients are within the hospital. It is expected that one of the areas with the greatest risk is the ER.

\section{The emergency room $\&$ the increase in carbapenem resistance}

The ER is an area in which patients are submitted to invasive procedures, usually in an urgent situation. Sometimes patient stretchers are closer to each other than recommended, and compliance with hand hygiene is worse than in other wards. Often the healthcare workers in the ER have a high workload, with frequent understaffing, and with high professional turnover rate [14]. Considering this context, cohorting patients and assigning dedicated staff may be a strategy to reduce transmission [15].

A study in a Brazilian ER described that $20 \%$ of patients with prolonged ER hospitalization (greater than 1 week) became colonized with CRE, demonstrating that this population is at high risk for colonization [16].

Screening patients, early isolation and contact precautions for CRE-colonized patients in the ER may have an impact on controlling colonization and transmission of CRE not only in the ER but in other areas of the hospital [17].

One important pitfall for controlling CRE in ER is how long it takes to obtain results of cultures. Patients should not remain in the ER for long periods of time. Culturing is often not feasible in this situation. Using other nonculture methods to screen patients, such as real-time PCR from rectal swabs, reduces the turnaround time for results and is an effective but expensive technology. One point against surveillance using real-time PCR is its inability to detect new mechanisms of resistance. Therefore, this strategy should be used in very specific contexts.

A possible strategy could be universal screening of patients in the ER during a limited amount of time, if the ER is considered an important source of CRE spread to other areas of the hospital. However, the causes of 
crosstransmission should also be investigated and resolved. In situations where the ER is responsible for a small but constant number of new cases of CRE, screening high risk patients is a possibility.

Therefore, we believe that surveillance cultures should be recommended in high-risk units for CRE, such as ICUs and wards with immunocompromised patients; and should be performed in countries with high or moderate CRE prevalence. However, in a situation of uncontrolled CRE dissemination, we need to think out of the box and look for possible caveats and unexpected sources. In this scenario, patients coming from community colonized or infected by CRE are becoming less of an unpredictable event and more of an increasing possibility.

\section{Future perspectives}

The CRE-colonized patients in hospitals are the tip of the iceberg of a much larger problem. The fact that we can detect carbapenemases coming from community settings, from patients in the ER, food, animals and others sources, demonstrates that this issue is no longer restricted to the hospitals, but to a much broader transmission network. Studies should be addressed to understand it. We believe in an overlapping network of transmission described in the concept of One Health. All efforts put into hospital control of transmission of CRE, such as creating strategies that are efficient, sensitive and cost effective for early CRE identification, will be partly efficacious. The fact that carbapenem resistance is no longer restricted to the hospital makes CRE control more challenging.

In the past, extended spectrum beta-lactamase-producing Enterobacteriaceae successfully spread to the community and now are frequently indistinguishable from hospital-bourne strains [18,19]. It would seem that at one point healthcare-associated extended spectrum beta-lactamase passed the barrier into the community. Most patients in the community are not colonized by CRE, but the ones who present risk factors, such as repeat infections, previous antibiotics use, previous hospitalization and residing in a nursing home are more prone to becoming colonized. Probably, there is a middle stage in which multidrug resistant bacteria are already found outside the hospital but have not disseminated enough to be a visible cause of CAI.

Because of all these considerations, many questions require answers in order to improve the identification of patients in the community who are at risk for CRE infection/colonization. Some of these questions are: how long does a patient remain colonized by CRE; what type of patients remain colonized for long periods of time and which ones will develop infections; which procedures or treatments lead to CRE infection or prolonged colonization; and, which are the outpatients services that are potential sources for CRE and in which interventions may have an impact and be cost effective? Meanwhile, strategies need to be focused on the hospitals. The ER, as an important intersection between the hospital and the community, should be included as a crucial part of the process.

\section{Author contributions}

MC Salomão and MP Freire wrote the manuscript. ASS Levin reviewed the manuscript.

\section{Financial \& competing interests disclosure}

The authors have no relevant affiliations or financial involvement with any organization or entity with a financial interest in or financial conflict with the subject matter or materials discussed in the manuscript. This includes employment, consultancies, honoraria, stock ownership or options, expert testimony, grants or patents received or pending, or royalties.

No writing assistance was utilized in the production of this manuscript.

\section{References}

1. Castanheira M, Deshpande LM, Mendes RE, Canton R, Sader HS, Jones RN. Variations in the occurrence of resistance phenotypes and carbapenemase genes among Enterobacteriaceae isolates in 20 years of the SENTRY antimicrobial surveillance program. Open Forum Infect. Dis. 6(Suppl. 1), S23-S33 (2019).

2. Friedman ND, Carmeli Y, Walton AL, Schwaber MJ. Carbapenem-resistant Enterobacteriaceae: a strategic roadmap for infection control. Infect. Control Hosp. Epidemiol. 38(5), 580-594 (2017).

3. Potter RF, D’Souza AW, Dantas G. The rapid spread of carbapenem-resistant Enterobacteriaceae. Drug Resist. Updat. 29, 30-46 (2016).

4. Dalben MDF, Teixeira Mendes E, Moura ML et al. A model-based strategy to control the spread of carbapenem-resistant Enterobacteriaceae: simulate and implement. Infect. Control Hosp. Epidemiol. 37(11), 1315-1322 (2016).

5. Caltagirone M, Nucleo E, Spalla M et al. Occurrence of extended spectrum $\beta$-lactamases, KPC-type, and MCR-1.2-producing Enterobacteriaceae from wells, river water, and wastewater treatment plants in Oltrepò Pavese area, northern Italy. Front. Microbiol. 8(2232), 1-12 (2017). 
6. Abdallah HM, Alnaiemi N, Reuland EA et al. Fecal carriage of extended-spectrum $\beta$-lactamase- and carbapenemase-producing Enterobacteriaceae in Egyptian patients with community-onset gastrointestinal complaints: a hospital-based cross-sectional study. Antimicrob. Resist. Infect. Control. 6(1), 1-7 (2017).

7. Zurfluh K, Poirel L, Nordmann P, Klumpp J, Stephan R. First detection of Klebsiella variicola producing OXA-181 carbapenemase in fresh vegetable imported from Asia to Switzerland. Antimicrob. Resist. Infect. Control. 4(1), 1-3 (2015).

8. Bartley PS, Domitrovic TN, Moretto VT et al. Antibiotic resistance in Enterobacteriaceae from surface waters in urban Brazil highlights the risks of poor sanitation. Am. J. Trop. Med. Hyg. 100(6), 1369-1377 (2019).

9. Khan FA, Hellmark B, Ehricht R, Söderquist B, Jass J. Related carbapenemase-producing Klebsiella isolates detected in both a hospital and associated aquatic environment in Sweden. Eur. J. Clin. Microbiol. Infect. Dis. 37(12), 2241-2251 (2018).

10. Kelly AM, Mathema B, Larson EL. Carbapenem-resistant Enterobacteriaceae in the community: a scoping review. Int. J. Antimicrob. Agents 50(2), 127-134 (2017).

11. Schwaber MJ, Lev B, Israeli A et al. Containment of a country-wide outbreak of carbapenem-resistant Klebsiella pneumoniae in Israeli hospitals via a nationally implemented intervention. Clin. Infect. Dis. 52(7), 848-55 (2011)

12. Lee BY, Bartsch SM, Hayden MK et al. How introducing a registry with automated alerts for carbapenem-resistant Enterobacteriaceae (CRE) may help control CRE spread in a region. Clin. Infect. Dis. doi:doi.org/10.1093/cid/ciz300 (2019) (Epub ahead of print).

13. Arena F, Vannetti F, Di Pilato V et al. Diversity of the epidemiology of carbapenemase-producing Enterobacteriaceae in long-term acute care rehabilitation settings from an area of hyperendemicity, and evaluation of an intervention bundle. J. Hosp. Infect. 100(1), 29-34 (2018).

14. Carter EJ, Wyer P, Giglio J et al. Environmental factors and their association with emergency department hand hygiene compliance: an observational study. BMJ Qual. Saf. 25(5), 372-328 (2016).

15. Legeay $\mathrm{C}$, Thépot-Seegers $\mathrm{V}$, Pailhoriès $\mathrm{H}$, Hilliquin $\mathrm{D}$, Zahar J-R. Is cohorting the only solution to control carbapenemase-producing Enterobacteriaceae outbreaks? A single-centre experience. J. Hosp. Infect. 99(4), 390-395 (2018).

16. Salomão MC, Guimarães T, Duailibi DF et al. Carbapenem-resistant Enterobacteriaceae in patients admitted to the emergency department: prevalence, risk factors, and acquisition rate. J. Hosp. Infect. 97(3), 241-246 (2017).

17. Munoz-Price LS, Quinn JP. Deconstructing the infection control bundles for the containment of carbapenem-resistant Enterobacteriaceae. Curr. Opin. Infect. Dis. 26(4), 378-387 (2013).

18. Kim YA, Kim JJ, Kim H, Lee K. Community-onset extended-spectrum-b-lactamase-producing Escherichia coli sequence type 131 at two Korean community hospitals: the spread of multidrug-resistant $E$. coli to the community via healthcare facilities. Int. J. Infect. Dis. 54, 39-42 (2017)

19. Pitout JDD, Laupland KB. Extended-spectrum $\beta$-lactamase-producing Enterobacteriaceae: an emerging public-health concern. Lancet Infect. Dis. 8(3), 159-166 (2008). 